\title{
Support E-Science Activities in Indonesia
}

\author{
Basuki Suhardiman ${ }^{1}$ \\ Institut Teknologi Bandung \\ Jalan Tamansari No. 64 Bandung, Indonesia \\ E-mail: basukieitb.ac.id
}

E-Science activities in Indonesia started in 2006 with development of the Indonesia Higher Education and Research Network (INHERENT). One of the activities was developing Grid Computing performed by several universities in Indonesia. The INGRID (Indonesia Grid) initiative started with the University of Indonesia. The development continued when ITB joined EUAsiaGrid Project and connected Indonesia Grid to International Grid Infrastructure. Some research to port existing application has been conducted but not yet able to deliver the expected result.

With the limited resources in Indonesia, several university initiate E-science activities for specific areas such as Weather prediction model, Computational Chemistry, Bio-informatics and Disaster Mitigation. Weather prediction model using MM5 and WrF model could only cover Java Area in Indonesia with the resolution $27 \mathrm{~km}$ x $27 \mathrm{~km}$, but could upgrade to $9 \mathrm{~km} \times 9 \mathrm{~km}$ using grid infrastructure. The Computational Chemistry is using several applications such as ABINIT, Gaussian and V-Sim. The Bio-informatics is using BLAST as their mail application. The other initiative supports activities for the Disaster Mitigation using information from the several government sources.

Development of training also has been delivered for specific requirements. Trainings focused on empowering the human resource that are able to run and administering grid infrastructure for their own activities.

Keyword: Grid, weather prediction, Computational chemistry, Disaster Mitigation, Bioinformatics.

The International Symposium on Grids and Clouds (ISGC) 2012

Academia Sinica, Taipei, Taiwan

February 26-March 2, 2012

\footnotetext{
1 Speaker
} 


\section{Introduction}

The development of Indonesia Higher Education and Research Network (INHERENT) in 2006 brought the concept of sharing the resources, including computing resources, of the Indonesian's Universities and Institutes. This underlies the development of e-science in Indonesia.

University of Indonesia (UI) created an opening to e-Science by building Grid Infrastructure called INGRID (Indonesia Grid) that is available for all university in Indonesia connected to INHERENT. However, development of INGRID was constrained by the network infrastructure and hardware capacity as well as finding suitable application for existing users.

Institute of Technology Bandung (ITB) broaden the scope when it joined EUAsiaGrid and connecting Indonesia Grid to International Grid Infrastructure. Building local Grid Sites, porting local application to be available on Grid, and dissemination of information about Grid system are the main activities undertaken to support e-science activities in Indonesia. The support for eScience activities in Indonesia is mainly lead by ITB.

In addition, Indonesia is one of the TEIN3 (Trans-Eurasia Information Network Phase 3) partners in Asia-Pacific region with ITB as the representative. TEIN3 provides high capacity internet network especially for research and education. The collaboration opens the opportunity to connect and share resources with other universities and institution connected to the same network. While INHERENT connects universities in Indonesia, TEIN3 bring the connection further to the world.

ITB has joined the Desktop Grid Initiatives with International Desktop Grid Federation (IDGF). The idea using Desktop Grid is to utilize the idle capacity on CPU in the Desktop. In ITB, there are more than 5000 computers connected in which about 3000 of them can be used. It means more than 3000 cores estimated. This is opportunity that can be utilized.

\section{Grid Infrastructure}

When ITB joined EUAsiaGrid, ITB built a Grid Site called ID-ITB that was connected to EUAsiaGrid Infrastructure. This site is currently being rebuilt to upgrade the installed services. The currently installed services are gapel.grid.itb.ac.id, gtorque.grid.itb.ac.id, glitewms.grid.itb.ac.id, glite-se.grid.itb.ac.id, and glite-ce.grid.itb.ac.id. The site is not yet released for production, because it still in the development and testing stage and will be used for application porting purposes.

In addition to the ID-ITB grid site, there are also some GPU clusters available. These GPU clusters are projected to be integrated to ID-ITB site. However, these GPU cluster are currently used by some research groups in ITB, mainly Weather Prediction and Computational Chemistry, to perform their research and work that needs high computational resources.

Last year, ITB attempt to expand its Grid Infrastructure with Desktop Grid. With knowledge from Desktop Grid seminar and small workshop in ITB by Dr. Robert Lovas, BOINC server for desktop Grid in ITB have successfully established with 4Gbytes of RAM. Applications are ready to be installed in this BOINC server. BOINC client was able to connect 
to the server and request a task/workunits to the server. However, there are still some problems for running application based on BOINC; the task cannot be processed by the clients. This could be due to some problems with the application porting. Apparently, some support actions are still needed in this area.

\section{Application Support}

Applications used by e-Science communities which run on clusters are planned to be ported to be available on grid infrastructure. The existing activity is an experiment to port Weather Prediction Application using WRF4G.

The Weather Prediction activity itself is currently working on improving their application and doing some experimental weather forecast extensions. Improvement in weather prediction application is modifying the resolution of the data input from $1^{0}$ (interval prediction every 6 hours) to $0.5^{0}$ (interval prediction every 3 hours). This means larger download volume but with (roughly) the same latency. The Grid infrastructure is used to support Numerical Weather Prediction (NWP) research activity which is concerned with the development of a common regional platform for NWP application in Southeast Asia. NWP experiment has been performed by implementing WRF4G (WRF for Grid) developed by University of Cantabria under EELA2 to find the most suitable downscaling strategy for NWP in South East Asia.

Beside the main weather prediction activity, there are also some experimental weather forecast extensions. Experimental weather forecast extension consist of volcanic ash distribution prediction, using tropical belt as prediction domain, and assimilation of radar data for highresolution local prediction.

Volcanic ash distribution prediction aims to quick forecasting volcanic ash dispersion up to 24 hours after a volcanic eruption. Computational resources needed to perform quick processing of available satellite data in near real-time, calculating volcanic ash trajectory using PUFF model (up to a million particle), and do iterative process to estimate the height of volcanic ash plume. Downloading large volume satellite data for near real-time result is supported by TEIN3 Network. Calculation and iterative processes can be supported by Grid Infrastructure. This experimental application is currently analyzed to be developed in cluster system and projected to be ported to grid infrastructure.

Prediction for tropical belt domain aims to provide downscaled weather prediction for the whole tropics with $27 \mathrm{~km}$ horizontal grid resolution suitable for middle-range (up to one week) weather forecasting and also to establish a common platform for collaborative research in tropical weather forecasting using WRF model. Significant amount of computer memory and CPU cores is required to perform this prediction and ensemble prediction for middle-range weather forecast.

Radar data assimilation aims to develop a prototype system of radar data assimilation for local weather forecasting. Large bandwidth is required for data transfer in near real time implementation, while huge amount of memory and CPU cores required to perform local prediction which uses $3 \mathrm{~km}$ grid resolution. 
The large data transfer or download for e-Science applications are supported by TEIN3 and INHERENT Network. Current large scale computations are using clusters and GPU clusters, and are mainly for weather prediction activity including the experimental extensions.

\section{Dissemination}

Dissemination activity is important to spread the knowledge of grid infrastructure so that more people involved in its development. Both in the development of infrastructure and applications. The dissemination can be in form of articles published in websites for reference, conference for knowledge sharing, or trainings to produce skilled personnel.

The latest dissemination activity held in ITB is seminar on Desktop Grid Computing, with Dr. Robert Lovas from IDGF as main speaker and M. Farhan S. From UPM as speaker. As expected, this event has brought more people involved in grid computing, especially in Desktop Grid.In the future, it is expected that there will be more events with speakers from university or institution that already have grid infrastructure running so that they can share best practices they used to the participants.

\section{Conclusions}

Support for e-Science activities in Indonesia can still be optimized. Existing resources can be utilized as part of the grid infrastructure. The limitation is in human resources with expertise grid computing system in Indonesia. In fact, the expertise is the major requirement in providing full support for e-Science. Therefore, activities that aim to provide knowledge and expertise in managing Grid Computing need to be held more intensive.

\section{References}

[1] B. Suhardiman, Grids and Clouds in ITB, in proceedings of ISGC $2011 \&$ OGF 31 PoS (ISGC $2011 \&$ OGF 31) 102. 